\title{
CORRELATIONS BETWEEN EXCESS OF LOSS REINSURANCE COVERS AND REINSURANCE OF THE $n$ LARGEST CLAIMS
}

\section{BARUCH BERLINER \\ Zurich}

E. Franckx [I] has established the distribution function of the largest individual claim of a portfolio. By assuming the number of claims to be Poisson distributed, $\mathrm{H}$. Ammeter was able to develop the distribution function of the total loss excluding the largest individual claim [2] as well as the distribution function of the $n^{\text {th }}$ largest claim [3].

Of course, the $n^{\text {th }}$ largest claim is dependent on the largest claim, second largest claim and so on, down to the $\left(n^{\text {th }}-\right.$ I) largest claim. If we assume the number of claims to be Poisson distributed and the amount of the individual claim to be Pareto distributed, the correlation between the $m^{\text {th }}$ largest and the $n^{\text {th }}$ largest claim can be expressed by an analytical formula which is susceptible to numerical computation.

With this knowledge we shall be able to compute the variance of the sum of the $n$ largest claims and moreover the correlation between the sum of the $n$ largest claims and the total loss amount. Although an excess of loss reinsurance treaty and a treaty reinsuring the $n$ largest claims are very different in their construction, this paper will show that from a practical point of view there exists a similarity between the two treaties. The correlation coefficient between the sum of the $n$ largest claims and the sum of all claims exceeding a certain limit enables us to assess the degree of similarity.

The correlation coefficient and thus the degree of similarity will prove to be high even in case of the reinsurance of only a small number of largest claims.

Finally, the knowledge of the two first moments of the sum of the $n$ largest claims allows us to compute the premium and the security or variance loading for the reinsurance of the $n$ largest claims. 
The methods applied in the following can also be used for the calculation of higher moments of the distribution function of the sum of the $n$ largest claims.

A. The expected value of the product of the $m^{\text {th }}$ and the $n^{\text {th }}$ largest claim

Let $x_{n}$ be the $n^{\text {th }}$ largest claim, $t$ the expected number of claims and $F(x)$ the distribution function of the individual claim amount. All the following formulae will be based on the Poisson risk process. The probability of the largest claim being smaller than $x$ is equal to the probability that all claims are smaller than $x$. Thus the distribution function of the largest claim in the Poisson case is:

$$
\phi_{1}(x)=\sum_{r=0}^{\infty} \frac{e^{-t} t^{r}}{r !}(F(x))^{r}=e^{-t(1-F(x))}
$$

The probability of the second largest claim being smaller than $x$ is equal to the probability of all claims being smaller than $x$ plus the probability of all but one claim being smaller than $x$. Thus

$$
\begin{aligned}
& \phi_{2}(x)=\sum_{r=0}^{\infty} \frac{e^{-t} t^{r}}{r !}(F(x))^{r}+(\mathrm{I}-F(x)) \sum_{r=1}^{\infty} r \frac{e^{-t} t^{r}}{r !}(F(x))^{r-1}= \\
&=\sum_{r=0}^{\infty} \frac{e^{-t} t^{r}}{r !}(F(x))^{r}+t(\mathrm{I}-F(x)) \sum_{r=0}^{\infty} \frac{e^{-t} t^{r}}{r !}(F(x))^{r} \\
&=(\mathrm{I}+t(\mathrm{I}-F(x))) e^{-t(1-F(x))} \\
& \phi_{n}(x)=\{\mathrm{I}+t(\mathrm{I}-F(x))+ \\
&\left.+\frac{t^{2}}{2 !}(\mathrm{I}-F(x))^{2}+\ldots+\frac{t^{n-1}}{(n-\mathrm{I}) !}(\mathrm{I}-F(x))^{n-1}\right\} \cdot e^{-t(1-F(x))}
\end{aligned}
$$

The probability of the largest claim being smaller than $x$ and of the second largest claim being smaller than $y$ is:

$\phi_{12}(x, y)=\sum_{r=0}^{\infty} \frac{e^{-t} t^{r}}{r !}(F(y))^{r}+(F(x)-F(y)) \sum_{r=1}^{\infty} r \frac{e^{-t} t^{r}}{r !}(F(y))^{r-1}=$ 


$$
\begin{aligned}
& =e^{-t(1-F(y))}(\mathrm{I}+t(F(x)-F(y))) \\
\phi_{13}(x, y)= & e^{-t(1-F(y))}\left(\mathrm{I}+t(F(x)-F(y))+\frac{t^{2}}{2 !}(F(x)-F(y))^{2}\right) \\
\phi_{23}(x, y)= & \sum_{r=0}^{\infty} \frac{e^{-t} t^{r}}{r !}(F(y))^{r}+\sum_{r=1}^{\infty} r(\mathrm{I}-F(y)) \frac{e^{-t} t^{r}}{r !}(F(y))^{r-1}+ \\
+ & \frac{\mathrm{I}}{2 !} \sum_{r=2}^{\infty} r(r-\mathrm{I})(F(x)-F(y))^{2} \frac{e^{-t} t^{r}}{r !}(F(y))^{r-2}+ \\
+ & \sum_{r=2}^{\infty} r(r-\mathrm{I})(\mathrm{I}-F(x))(F(x)-F(y)) \frac{e^{-t} t^{r}}{r !}(F(y))^{r-2}= \\
= & e^{-t(1-F(y))}\left\{\mathrm{I}+t(\mathrm{I}-F(y))+\frac{t^{2}}{2 !}(F(x)-F(y))^{2}+\right. \\
& \left.\quad+t^{2}(\mathrm{I}-F(x))(F(x)-F(y))\right\}
\end{aligned}
$$

Following the same line of thought as suggested in the three previous formulae we arrive at the following general formula:

$$
\begin{aligned}
& \phi_{m, n}(x, y)=\phi_{m, n-1}(x, y)+ \\
&+\frac{t^{n-1}}{(n-\mathrm{I}) !} \sum_{\nu=0}^{m-1}\left(\begin{array}{c}
\mathrm{n}-\mathrm{I} \\
\nu
\end{array}\right)(\mathrm{I}-F(x))^{\nu}(F(x)-F(y))^{n-1-\nu}= \\
&=\phi_{m, n-1}(x, y)+\frac{t^{n-1}}{(n-\mathrm{I}) !} \cdot\left\{(\mathrm{I}-F(y))^{n-1}-\right. \\
&\left.-\sum_{v=m}^{n-1}\left(\begin{array}{c}
\mathrm{n}-\mathrm{I} \\
v
\end{array}\right)(\mathrm{I}-F(x))^{\nu}(F(x)-F(y))^{n-1-\nu}\right\} \\
& \text { where } \quad n>m .
\end{aligned}
$$

For $m=\mathrm{I}$ we get

$$
\begin{aligned}
\phi_{1, n}(x, y) & =\phi_{1, n-1}(x, y)+\frac{t^{n-1}}{(n-\mathrm{I}) !}(F(x)-F(y))^{n-1}= \\
& =e^{-t(1-F(y))}\{\mathrm{I}+t(F(x)-F(y))+\cdots+ \\
& \left.+\frac{t^{n-1}}{(n-\mathrm{I}) !}(F(x)-F(y))^{n-1}\right\}
\end{aligned}
$$


For $m=n-\mathrm{I}$ we get

$$
\begin{aligned}
\phi_{n-1, n}(x, y)=\phi_{n-1}(y)+\frac{t^{n-1}}{(n-\mathrm{I}) !}\left\{(\mathrm{I}-F(y))^{n-1}-\right. \\
\left.-(\mathrm{I}-F(x))^{n-1}\right\}
\end{aligned}
$$

We now want to calculate the probability density $\varphi_{m, n}$.

$$
\phi_{12}(x, y)=\int_{0}^{y} \int_{0}^{v} \varphi_{12}(u, v) d u d v+\int_{y}^{x} \int_{0}^{y} \varphi_{12}(u, v) d u d v .
$$

The first function on the right hand side is equal to the probability that the largest claim will be smaller than $y$. From (I) and (3) we can thus derive the two conditions

$$
\begin{aligned}
& \int_{0}^{y} \int_{0}^{0} \varphi_{12}(u, v) d u d v=e^{-t(1-F(y))} \\
& \int_{y}^{x} \int_{0}^{y} \varphi_{12}(u, v) d u d v=t(F(x)-F(y)) e^{-t(1-F(y))}
\end{aligned}
$$

The two conditions are satisfied by

$$
\varphi_{12}(u, v)=t^{2} f(v) f(u) e^{-t(1-F(u))} \quad \text { where } f(u)=\frac{d F(u)}{d u}
$$

as can easily be verified.

$\varphi_{13}(u, v)$ has to satisfy the following three conditions:

$$
\begin{aligned}
\int_{0}^{y} \int_{0}^{w} \int_{0}^{w} \varphi_{13}(u, v, w) d u d v d w & =e^{-t(1-F(y))} \\
\int_{y}^{x} \int_{0}^{y} \int_{0}^{v} \varphi_{13}(u, v, w) d u d v d w & =t(F(x)-F(y)) e^{-t(1-F(y))} \\
\int_{\nu}^{x} \int_{\nu}^{w} \int_{0}^{y} \varphi_{13}(u, v, w) d u d v d w & =\left(t^{2} / 2 !\right)(F(x)-F(y))^{2} e^{-t(1-F(y))} \\
\varphi_{13}(u, v, w) & =t^{3} f(w) f(v) f(u) e^{-t(1-F(u))}
\end{aligned}
$$

satisfies the three conditions listed above.

The general formula runs as follows:

$$
\begin{aligned}
\varphi_{m n}\left(u_{1}, u_{2}, \ldots, u_{n}\right) & =\varphi_{1 n}\left(u_{1}, u_{2}, \ldots, u_{u}\right)=\varphi_{12} \cdots n\left(u_{1}, u_{2}, \ldots u_{n}\right)= \\
& =t^{n} \prod_{j=1}^{n} f\left(u_{j}\right) e^{-t\left(1-F\left(u_{1}\right)\right)}, \text { where } m<n
\end{aligned}
$$


From now on we shall assume that the individual claim amount is Pareto distributed.

$$
\begin{gathered}
\left.f(x)=\alpha x^{-\alpha-1} \quad \mathrm{I} \leqslant x<\infty\right) \\
F(x)=\mathrm{I}-x^{-\alpha} \quad(\mathrm{I} \leqslant x<\infty) \\
\varphi_{m n}\left(u_{1}, u_{2}, \ldots, u_{n}\right)=t^{n} \alpha^{n} \prod_{j=1}^{n} u_{j}^{-\alpha-1} e^{-t u^{-\alpha}}
\end{gathered}
$$

Now we are able to compute the expected value

$$
E\left(x_{m} x_{n}\right)
$$

In order to illustrate the procedure we shall again start with the simplest case, namely: $E\left(\begin{array}{ll}x_{1} & x_{2}\end{array}\right)$

$$
\begin{aligned}
E\left(x_{1} x_{2}\right) & =\int_{1}^{\infty} \int_{1}^{0} u v \varphi_{12}(u, v) d u d v= \\
& =\alpha^{2} t^{2} \int_{1}^{\infty} \int_{1}^{v} u^{-\alpha} v^{-\alpha} e^{-t u^{-\alpha}} d u d v
\end{aligned}
$$

By substituting $x=t u^{-\alpha}, y=t v^{-\alpha}$ we obtain

$$
\begin{gathered}
E\left(x_{1} x_{2}\right)=t^{2 / \alpha} \int_{0}^{t} \int_{y}^{t} e^{-x} x^{(\alpha-1 / \alpha)-1} y^{-1 / \alpha} d x d y= \\
=t^{2 / \alpha} \int_{0}^{t}\left[\Gamma\left(\frac{\alpha-\mathrm{I}}{\alpha}\right)-\Gamma\left(\frac{\alpha-\mathrm{I}}{\alpha}\right)\right] y^{-1 / \alpha} d y= \\
=t^{2 / \alpha}\left\{\Gamma\left(\frac{\alpha-\mathrm{I}}{\alpha}\right) \frac{\alpha}{\alpha-\mathrm{I}} t^{(\alpha-1 / \alpha)}-\frac{\alpha}{\alpha-\mathrm{I}} t^{(\alpha-1 / \alpha)} \Gamma \frac{\alpha-\mathrm{I}}{\alpha}+\right. \\
\left.+\frac{\alpha}{\alpha-\mathrm{I}} \Gamma_{t}\left(\frac{2 \alpha-2}{\alpha}\right)\right\} \\
E\left(x_{1} x_{2}\right)=t^{2 / \alpha} \frac{\alpha}{\alpha-\mathrm{I}} \Gamma\left(\frac{2 \alpha-2}{\alpha}\right)
\end{gathered}
$$

The extensive calculation leading to the general formulae are omitted.

$$
E\left(x_{1} x_{n}\right)=t^{2 / \alpha} \prod_{j=1}^{n-1} \frac{\alpha}{j \alpha-\mathrm{I}} \Gamma_{!}\left(\frac{n \alpha-2}{\alpha}\right)
$$




$$
\begin{gathered}
E\left(x_{m} x_{n}\right)=\frac{t^{2 / \alpha}}{(m-\mathrm{I}) !} \prod_{j=m}^{n-1} \frac{\alpha}{j \alpha-\mathrm{I}} \Gamma\left(\frac{n \alpha-2}{\alpha}\right)= \\
=\frac{\mathrm{I}}{(m-\mathrm{I}) !} \prod_{j=1}^{m-1} \frac{j \alpha-\mathrm{I}}{\alpha} E\left(x_{1} x_{n}\right) \\
\text { where } m<n
\end{gathered}
$$

We also have

$$
E\left(x_{1} x_{1}\right)=\int_{1}^{\infty} t \alpha u^{-\alpha+1} e^{-t u^{-\alpha}} d u=t^{2 / \alpha} \Gamma\left(\frac{\alpha-2}{\alpha}\right)
$$

This formula has already been given by $H$. Ammeter [2] The general formula runs as follows

$$
E\left(x_{n} x_{n}\right)=t^{2 / \alpha} \frac{\mathrm{I}}{(n-\mathrm{I}) !} \Gamma\left(\frac{n \alpha-2}{\alpha}\right)
$$

For small $n$ and not too small $t$ the incomplete Gamma functions in (II), (I2), (I3) and (I4) can be replaced by complete Gamma functions.

If we take as an example $\alpha=3$ and $t=\mathrm{Io}$, then we produce for (Example I)

$$
\begin{aligned}
& n=2: \Gamma\left(\frac{n \alpha-2}{\alpha}\right) / \Gamma\left(\frac{n \alpha-2}{\alpha}\right)=0,9999 \\
& n=4: \Gamma\left(\frac{n \alpha-2}{\alpha}\right) / \Gamma\left(\frac{n \alpha-2}{\alpha}\right)=0,9955 \\
& n=\Gamma_{t}: \Gamma_{t}\left(\frac{n \alpha-2}{\alpha}\right) / \Gamma\left(\frac{n \alpha-2}{\alpha}\right)=0, \mathrm{I} 820 \\
& n=24: \Gamma\left(\frac{n \alpha-2}{\alpha}\right) / \Gamma\left(\frac{n \alpha-2}{\alpha}\right)=0,0002
\end{aligned}
$$

When using complete Gamma functions in (I4) and (I2) we arrive at:

$$
E\left(x_{n} x_{n}\right) \approx \frac{\mathrm{I}}{n-\mathrm{I}} \frac{(n-\mathrm{I}) \alpha-2}{\alpha} E\left(x_{n-1} x_{n-1}\right)
$$




$$
\begin{aligned}
E\left(x_{m}, x_{n}\right) & \approx \frac{(n-\mathrm{I}) \alpha-2}{(n-\mathrm{I}) \alpha-\mathrm{I}} E\left(x_{m} \cdot x_{n-1}\right) \\
& \approx \frac{\mathrm{I}}{m-\mathrm{I}} \frac{(m-\mathrm{I})}{\alpha} \frac{\alpha-\mathrm{I}}{} E\left(x_{m-1} x_{n}\right)
\end{aligned}
$$

where $m<n$

Formulae (I5) and (I6) are exact equations in the limiting case for $t \rightarrow \infty$.

When deriving the formulae (II), (I2) and (I3) we obtained the following interesting and useful identities:

$$
\begin{aligned}
& \int_{1}^{\infty} \int_{1}^{u_{1}} \int_{1}^{n_{2}} \int_{1}^{u_{n-1}} u_{1}^{-\alpha-1} u_{2}^{-\alpha-1} \ldots u_{n-1}^{-\alpha-1} u_{n}^{-\alpha+1} e^{t u_{n}^{-\alpha}} d u_{n} d u_{n-1} \ldots d u_{1} \\
& =t^{2 / \alpha} \int_{0}^{t} \int_{v_{1}}^{t} \int_{v_{2}}^{t} \ldots \int_{v_{n-1}}^{t} v_{n}^{-2 / \alpha} e^{-v_{n}} d v_{n} d v_{n-1} \ldots d v_{1} \\
& =t^{2 / \alpha} \frac{\mathrm{I}}{(n-\mathrm{I}) !} \int_{0}^{t} e^{-v_{v}((n-1) \alpha-2) / \alpha} d v \\
& \int_{1}^{\infty} \int_{1}^{u_{1}} \int_{1}^{u_{2}} \ldots \int_{1}^{u_{n-1}} u_{1}^{-\alpha-1} \ldots u_{m-1}^{-\alpha-1} u_{m}^{-\alpha} u_{m+1}^{-\alpha-1} \ldots \\
& \ldots u_{n-1}^{-\alpha-1} u_{n}^{-\alpha} e^{-t u_{n}^{-\alpha}} d u_{n} d u_{n-1} \ldots d u_{1}= \\
& =t^{2 / \alpha} \int_{0}^{t} \int_{v_{1}}^{1} \int_{v_{2}}^{t} \ldots v_{m}^{-1 / \alpha} v_{n}^{-1 / \alpha} e^{-v_{n}} d v_{n} d v_{n-1} \ldots d v_{1}= \\
& =t^{2 / \alpha} \frac{\mathrm{I}}{(m-\mathrm{I}) !} \prod_{n-m}^{n-1} \frac{\alpha}{v \alpha-\mathrm{I}} \int_{0}^{t} e^{-v} v^{((n-1) \alpha-2) / \alpha} d v \text {, where } m<n
\end{aligned}
$$

Similar integral formulae can be developed when deriving expressions for higher moments of the sum of the $n$ largest claims.

B. The expected value and the variance of the sum of the $n$ largest claims

Calculating the derivative of $\phi_{n}$ in (2) we arrive at:

$$
\begin{gathered}
\varphi_{n}(x)=t f(x) e^{-t(1-F(x))} \frac{\{t(\mathrm{I}-F(x))\}^{n-1}}{(n-\mathrm{I}) !}= \\
=\alpha t x^{-\alpha-1} e^{-t x^{-\alpha}} \frac{\left(t x^{-\alpha}\right)^{n-1}}{(n-\mathrm{I}) !}
\end{gathered}
$$


From formula (rg), already given by $\mathrm{H}$. Ammeter [3], we can easily derive the expression for the average of the largest claim excess $A_{1}$ plus the average of the second largest claim excess $A_{2}$ and so on. For practical purposes it can be assumed that $A_{1} \geqslant A_{2} \geqslant \ldots$ $\geqslant A_{n}$

$$
\begin{gathered}
\sum_{i=1}^{n} E_{A_{i}}\left(x_{i}\right)=t^{1 / \alpha}\left[\Gamma_{t A_{1}-\alpha}\left(\frac{\alpha-\mathrm{I}}{\alpha}\right)+\frac{\mathrm{I}}{\mathrm{I} !} \Gamma_{t A_{2}-\alpha}\left(\frac{2 \alpha-\mathrm{I}}{\alpha}\right)+\ldots+\right. \\
\left.+\frac{\mathrm{I}}{(n-\mathrm{I}) !} \Gamma_{t A_{n}-\alpha}\left(\frac{n \alpha-\mathrm{I}}{\alpha}\right)\right]-\left[A_{1}\left(\mathrm{I}-e^{-t A_{1}-\alpha}\right)+\right. \\
\left.+\frac{A_{2}}{\mathrm{I} !} \Gamma_{t A_{2}-\alpha}(2)+\ldots+\frac{A_{n}}{(n-\mathrm{I}) !} \Gamma_{t A_{n}-\alpha}(n)\right]
\end{gathered}
$$

For very large $t$ the formula reduces to

$$
\sum_{i=1}^{n} E_{A_{i}}\left(x_{i}\right) \approx t^{1 / \alpha} \frac{\alpha}{\alpha-\mathrm{I}} \frac{\mathrm{I}}{(n-\mathrm{I}) !} \Gamma\left(\frac{(n+\mathrm{I}) \alpha-\mathrm{I})}{\alpha}\right)-\sum_{i=1}^{n} A_{i}
$$

the first term on the right hand side already having been mentioned by $\mathrm{H}$. Ammeter [3].

$$
\begin{gathered}
\sigma_{x_{1}+x_{2}+\cdots+x_{n}}^{2}= \\
=\sum_{i=1}^{n} E\left(x_{i}^{2}\right)-\sum_{i=1}^{n} E^{2}\left(x_{i}\right)+2 \sum_{i<j} E\left(x_{i} x_{j}\right)-2 \sum_{i<j} E\left(x_{i}\right) E\left(x_{j}\right)
\end{gathered}
$$

(22) can be computed for any $n$ from the formulae (r2), (14) and (20) respectively, and for small $n$ from the formulae (I5), (I6) and (20).

\section{Example II}

Let $n=3$

$$
\begin{aligned}
& \sigma_{x_{1}+x_{2}+x_{9}}^{2}=t^{2 / \alpha}\left\{\Gamma\left(\frac{\alpha-2}{\alpha}\right)+\Gamma\left(\frac{2 \alpha-2}{\alpha}\right)+\frac{I}{2 !} \Gamma\left(\frac{3 \alpha-2}{\alpha}\right)-\right. \\
& -\Gamma_{t}^{2}\left(\frac{\alpha-\mathrm{I}}{\alpha}\right)-\Gamma^{2}\left(\frac{2 \alpha-\mathrm{I}}{\alpha}\right)-\frac{\mathrm{I}}{(2 !)^{2}} \Gamma^{2}\left(\frac{3 \alpha-\mathrm{I}}{\alpha}\right)+
\end{aligned}
$$




$$
\begin{aligned}
& +2\left(\frac{\alpha}{\alpha-I}\right) \Gamma\left(\frac{2 \alpha-2}{\alpha}\right)+2 \frac{\alpha}{\alpha-I} \frac{\alpha}{2 \alpha-I} \Gamma\left(\frac{3 \alpha-2}{\alpha}\right)+ \\
& +2 \frac{\alpha}{2 \alpha-I} \Gamma\left(\frac{3 \alpha-2}{\alpha}\right)-2 \Gamma\left(\frac{\alpha-I}{\alpha}\right) \Gamma\left(\frac{2 \alpha-I}{\alpha}\right)- \\
& \left.-\frac{2}{2 !} \Gamma\left(\frac{\alpha-\mathrm{I}}{\alpha}\right) \Gamma\left(\frac{3 \alpha-\mathrm{I}}{\alpha}\right)-\frac{2}{2 !} \Gamma\left(\frac{2 \alpha-\mathrm{I}}{\alpha}\right) \Gamma\left(\frac{3 \alpha-\mathrm{I}}{\alpha}\right)\right\} \approx \\
& \approx t^{2 / \alpha}\left\{\left[( \frac { \alpha - 2 } { \alpha } ) \left[\mathrm{I}+\frac{\alpha-2}{\alpha}+\frac{\mathrm{I}}{2 !} \frac{\alpha-2}{\alpha} \frac{2 \alpha-2}{\alpha}+2 \frac{\alpha-2}{\alpha-\mathrm{I}}+\right.\right.\right. \\
& \left.+2 \frac{\alpha-2}{\alpha-I} \frac{2 \alpha-2}{2 \alpha-I}+2 \frac{2 \alpha-2}{2 \alpha-I} \frac{\alpha-2}{\alpha}\right]-\Gamma^{2}\left(\frac{\alpha-I}{\alpha}\right)\left[I+\left(\frac{\alpha-I}{\alpha}\right)^{2}+\right. \\
& +\frac{I}{4}\left(\frac{\alpha-I}{\alpha}\right)^{2}\left(\frac{2 \alpha-I}{\alpha}\right)^{2}+2 \frac{\alpha-I}{\alpha}+\frac{\alpha-I}{\alpha} \frac{2 \alpha-I}{\alpha}+ \\
& \left.\left.+\left(\frac{\alpha-I}{\alpha}\right)^{2} \frac{2 \alpha-I}{\alpha}\right]\right\} \\
& \alpha=2,5: \quad \sigma_{x_{1}+x_{2}+x_{3}}^{2} \approx 3, \mathrm{I} 985 t^{4 / s} \\
& \alpha=3,0: \quad \sigma_{x_{1}+x_{2}+x_{2}}^{2} \approx \mathrm{I}, 3630 t^{2 / 8} \\
& \alpha=4,0: \quad \sigma_{x_{1}+x_{2}+x_{3}}^{2} \approx 0,53696 t^{1 / 2}
\end{aligned}
$$

C. The correlation between the sum of the $n$ largest claims and the total loss amount

The expected value of the total loss is in the Poisson-Pareto case

$$
E=t \frac{\alpha}{\alpha-I}
$$

The variance of the total loss amounts to

$$
\begin{gathered}
\sigma^{2}=\frac{\alpha}{\alpha-2} t \\
P\left\{\left[x_{1}-E\left(x_{1}\right)+x_{2}-E\left(x_{2}\right)+\ldots+x_{n}-E\left(x_{n}\right)\right]\left[\sum_{i=1}^{\infty} x_{i}-\frac{\alpha}{\alpha-I} t\right]\right\} \\
\sigma_{x_{1}+x_{2}+\cdots+x_{n}} \sqrt{\frac{\alpha}{\alpha-2} t}
\end{gathered}
$$




$$
\begin{gathered}
=\frac{\sum_{i=1}^{n} E\left(x_{i}^{2}\right)-\sum_{i=1}^{n} E^{2}\left(x_{i}\right)+2 \sum_{i<j-1}^{n} E\left(x_{i} x_{j}\right)-2 \sum_{i<j-1}^{n} E\left(x_{i}\right) E\left(x_{j}\right)}{\sigma_{x_{1}+x_{2}+\cdots+x_{n}} \sqrt{\frac{\alpha}{\alpha-2} t}}+ \\
+\frac{\sum_{i=1,-n+1}^{n} \sum_{i}^{\infty} E\left(x_{i} x_{j}\right)-\sum_{i=1, j-n+1}^{n} E\left(x_{i}\right) E\left(x_{j}\right)}{\sigma_{x_{1}+x_{2}+\cdots+x_{n}} \sqrt{\frac{\alpha}{\alpha-2} t}} .
\end{gathered}
$$

From formula (22) follows

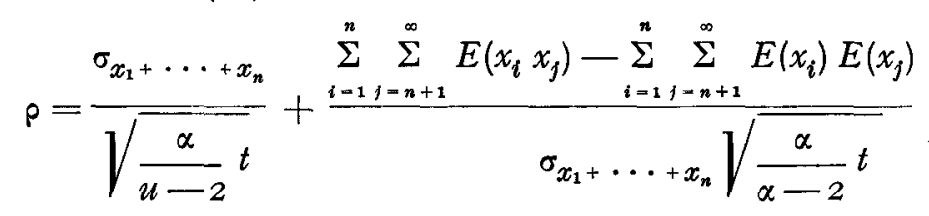

We can consider the infinite sums in (25) as limits of partial sums that are converging slowly. As can be learned from Example I, dozens of incomplete Gamma functions will usually have to be calculated if we want to determine $p$ according to formula (25) with reasonable accuracy.

We have, therefore, put some effort into developing equations which allow us to replace infinite sums of incomplete Gamma functions by other easily calculable functions. The only Gamma functions that we shall need for the calculation of $\rho$ will be those already needed in (22) for the determination of $\sigma_{x_{1}+x_{2}+\cdots x_{n}}$ (when $n \geqslant 3$ ). For small $n$, when the incomplete Gamma functions can be replaced by complete ones, the determination of two Gamma functions, as can be seen in Example II, is sufficient.

The expected value of the sum of all claims has to be identical with that of the total loss. Using formulae (20) and (23) we can thus write:

$$
\sum_{n=1}^{\infty} \frac{t^{1 / 2}}{(n-\mathrm{I}) !} \Gamma\left(\frac{n \alpha-\mathrm{I}}{\alpha}\right)=\frac{\alpha}{\alpha-\mathrm{I}} t \text {, where } \alpha>\mathrm{I}
$$

If we replace in $(26) \alpha$ by $\beta=\alpha / m>\mathrm{I}$ we arrive at:

$$
\sum_{n=1}^{\infty} \frac{t^{m / \alpha}}{(n-\mathrm{I}) !} \Gamma\left(\frac{n \alpha-m}{\alpha}\right)=\frac{\alpha}{\alpha-m} t, \text { where } \alpha>m
$$


For $m=2$ the right hand side of $(27)$ is equal to the variance of the total loss (compare (24)), whilst the left hand side of $(27)$ is equal for $m=2$, because of (I4), to the second moment about the origin of the sum of all claims.

Thus we can write:

$$
\begin{gathered}
\sum_{i=1}^{\infty} E\left(x_{i}^{2}\right)=E\left[\left(\sum_{i=1}^{\infty} x_{i}\right)^{2}\right]-\left[E\left(\sum_{i=1}^{\infty} x_{i}\right)\right]^{2} \leftrightarrow \\
\sum_{i<k=1}^{\infty} E\left(x_{i} x_{k}\right)=\frac{\mathrm{I}}{2}\left[E\left(\sum_{i=1}^{\infty} x_{i}\right)\right]^{2}=\frac{\mathrm{I}}{2}\left(\frac{\alpha t}{\alpha-\mathrm{I}}\right)^{2}
\end{gathered}
$$

H. Ammeter has established the distribution function of the total loss excluding the largest individual claim [2]. From this distribution function we can calculate the second moment around zero, with the following result:

$$
\begin{gathered}
E\left[\left(\sum_{i=2}^{\infty} x_{i}\right)^{2}\right]=\left[\left(\frac{t \alpha}{\alpha-\mathrm{I}}\right)^{2}+\frac{t \alpha}{\alpha-2}\right]\left(\mathrm{I}-e^{-t}\right)- \\
-2 \frac{\alpha^{2}}{(\alpha-\mathrm{I})^{2}} t^{(\alpha+1 / \alpha)} \Gamma_{t}\left(\frac{2 \alpha-\mathrm{I}}{\alpha}\right)+t^{\alpha / 2} \frac{\alpha^{2}}{(\alpha-\mathrm{I})^{2}} \Gamma_{i}\left(\frac{3 \alpha-2}{\alpha}\right)- \\
-t^{2 / \alpha} \frac{\alpha}{\alpha-2} \Gamma_{t}\left(\frac{2 \alpha-2}{\alpha}\right)=\frac{\alpha}{\alpha-2} t-t^{2 / \alpha} \Gamma_{t}\left(\frac{\alpha-2}{\alpha}\right)+ \\
+2 \sum_{i=2}^{\infty} E\left(x_{1} x_{i}\right)
\end{gathered}
$$

Combining (28) and (29) we arrive at the important equation:

$$
\begin{gathered}
\sum_{i=2}^{\infty} E\left(x_{1} x_{i}\right)=\frac{I}{2}\left\{\left[\left(\frac{t \alpha}{\alpha-\mathrm{I}}\right)^{2}+\frac{t \alpha}{\alpha-2}\right] e^{-t}-t^{2 / \alpha} \Gamma_{t}\left(\frac{\alpha-2}{\alpha}\right)+\right. \\
+2\left(\frac{\alpha}{\alpha-\mathrm{I}}\right)^{2} t^{(\alpha+1 / \alpha)} \Gamma_{t}\left(\frac{2 \alpha-\mathrm{I}}{\alpha}\right)-t^{2 / \alpha}\left(\frac{\alpha}{\alpha-\mathrm{I}}\right)^{2} \Gamma_{t}\left(\frac{3 \alpha-2}{\alpha}\right)+ \\
\left.+t^{2 / \alpha} \frac{\alpha}{\alpha-2} \Gamma\left(\frac{2 \alpha-2}{\alpha}\right)\right\}
\end{gathered}
$$


From (I2) we can derive

$$
\begin{gathered}
\sum_{i=k+1}^{\infty} E\left(x_{k} x_{i}\right)=\frac{\mathrm{I}}{k-\mathrm{I}} \frac{(k-\mathrm{I}) \alpha-\mathrm{I}}{\alpha} \sum_{i=k}^{\infty} E\left(x_{k-1} x_{i}\right)- \\
-t^{2 / \alpha} \frac{\mathrm{I}}{(k-2) !} \frac{\alpha}{(k-\mathrm{I}) \alpha-\mathrm{I}} \Gamma\left(\frac{k \alpha-2}{\alpha}\right)
\end{gathered}
$$

and

$$
\sum_{i=k+1}^{\infty} E\left(x_{l} x_{i}\right)=\frac{\mathrm{I}}{(l-\mathrm{I}) !} \prod_{i=1}^{l-1} \frac{j \alpha-\mathrm{I}}{\alpha} \sum_{i=k+1}^{\infty} E\left(x_{1} x_{i}\right) \text { for } l=2, \ldots, k
$$

For not too small $t$ we can replace the incomplete Gamma functions in (30) without any appreciable loss of accuracy by complete Gamma functions obtaining:

$$
\sum_{i=2}^{\infty} E\left(x_{1} x_{i}\right) \approx\left(\frac{\alpha}{\alpha-I}\right)^{2} t^{(\alpha+1 / \alpha)} \Gamma\left(\frac{2 \alpha-I}{\alpha}\right)-\frac{I}{2} t^{2 / \alpha} \frac{\alpha}{\alpha-I} \Gamma\left(\frac{2 \alpha-2}{\alpha}\right)
$$

Because of (26) we can now replace in (25)

$$
\begin{gathered}
\sum_{i=1}^{n} \sum_{j=n+1}^{\infty} E\left(x_{i}\right) E\left(x_{j}\right) \text { by } \\
\sum_{i=1}^{n} \sum_{j=n+1}^{\infty} E\left(x_{i}\right) E\left(x_{j}\right)=\left\{\sum_{i=1}^{n} \frac{t^{1 / \alpha}}{(i-\mathrm{I}) !} \Gamma\left(\frac{i \alpha-\mathrm{I}}{\alpha}\right)\right\} \\
\left\{\frac{\alpha}{\alpha-\mathrm{I}} t-\sum_{i=1}^{n} \Gamma\left(\frac{i \alpha-\mathrm{I}}{\alpha}\right)\right\}
\end{gathered}
$$

Because of (II), (30), (3I) and (32) we can replace

$$
\begin{gathered}
\sum_{i=1}^{n} \sum_{j=n+1}^{\infty} E\left(x_{i} x_{j}\right) \text { by } \\
\sum_{i=1}^{n} \sum_{j=n+1}^{\infty} E\left(x_{i} x_{j}\right)=\frac{\mathrm{I}}{2}\left\{\mathrm{I}+\sum_{j=1}^{n-1} \frac{\mathrm{I}}{j !} \prod_{i=1}^{1} \frac{i \alpha-\mathrm{I}}{\alpha}\right\}
\end{gathered}
$$




$$
\begin{aligned}
& \left\{\left[\left(\frac{t \alpha}{\alpha-\mathrm{r}}\right)^{2}+\frac{t \alpha}{\alpha-2}\right] e^{-t}+2\left(\frac{\alpha}{\alpha-\mathrm{I}}\right)^{2} t^{(\alpha+1 / \alpha)} \Gamma\left(\frac{2 \alpha-\mathrm{I}}{\alpha}\right)-\right. \\
& -t^{2 / \alpha} \Gamma_{t}\left(\frac{\alpha-2}{\alpha}\right)-t^{2 / \alpha}\left(\frac{\alpha}{\alpha-I}\right)^{2} \Gamma\left(\frac{3 \alpha-2}{\alpha}\right)+ \\
& \left.+t^{2 / \alpha}\left(\frac{\alpha}{\alpha-2}\right) \Gamma\left(\frac{2 \alpha-2}{\alpha}\right)-2 t^{2 / \alpha} \sum_{i=2}^{n} \prod_{i=1}^{t-1} \frac{\alpha}{i \alpha-I} \Gamma\left(\frac{i \alpha-2}{\alpha}\right)\right\}
\end{aligned}
$$

For small $n$, not too large $\alpha$ and not too small $t$, complete instead of incomplete Gamma functions can be used. Using (II), (2I), (25), (26), (32) and (33) we can then write:

$$
\begin{aligned}
& \rho(n, \alpha, t) \approx \frac{\sigma_{x_{1}+\ldots+x_{n}}}{\sqrt{\frac{\alpha}{\alpha-2} t}}+\left[\left\{I+\sum_{j=1}^{n-1} \frac{I}{j !} \prod_{i=1}^{1} \frac{i \alpha-I}{\alpha}\right\}\right. \\
& \left\{\left[\left(\frac{t \alpha}{\alpha-I}\right)^{2}+\frac{t \alpha}{\alpha-2}\right] e^{-t}+\left(\frac{\alpha}{\alpha-I}\right)^{2} t^{(\alpha+1 / \alpha)} \Gamma\left(\frac{2 \alpha-I}{\alpha}\right)-\right. \\
& \left.-t^{2 / \alpha} \Gamma\left(\frac{2 \alpha-2}{\alpha}\right) \frac{\alpha}{\alpha-I}\left[2+\sum_{i=3}^{n} \prod_{i=2}^{1-1} \frac{i \alpha-2}{i \alpha-I}\right]\right\}- \\
& -\left(\frac{\alpha}{\alpha-\mathrm{I}}\right)^{2}\left\{\frac{t^{1 / \alpha}}{(n-\mathrm{I}) !} \Gamma\left(\frac{(n+\mathrm{I}) \alpha-\mathrm{I}}{\alpha}\right)\right\}
\end{aligned}
$$

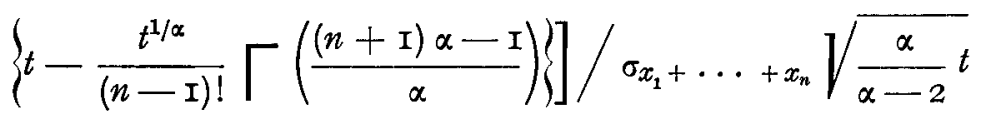

Because of the identity

$$
I+\sum_{j=1}^{n-1} \frac{I}{j !} \prod_{i=1}^{1} \frac{i \alpha-I}{\alpha}=\prod_{i=2}^{n} \frac{i \alpha-I}{(i-I) \alpha}
$$

(36) can be replaced by

$$
\rho(n, \alpha, t) \approx \frac{\sigma_{x_{1}+\cdots+x_{n}}}{\sqrt{\frac{\alpha}{\alpha-2} t}}+\left[\prod _ { i = 2 } ^ { n } \frac { i \alpha - I } { ( i - I ) \alpha } \left\{\left[\left(\frac{t \alpha}{\alpha-I}\right)^{2}+\right.\right.\right.
$$




$$
\begin{aligned}
& \left.+\frac{t \alpha}{\alpha-2}\right] e^{-t}-t^{2 / \alpha}\left[\left(\frac{2 \alpha-2}{\alpha}\right) \frac{\alpha}{\alpha-I}\left[2+\sum_{j=3}^{n} \prod_{i=3}^{i-1} \frac{i \alpha-2}{i \alpha-I}\right]\right\} \\
& +\left\{\frac{\alpha}{\alpha-I} \frac{t^{1 / \alpha}}{(n-I) !}\left[\left(\frac{(n+I) \alpha-I}{\alpha}\right)\right\}^{2}\right] / \sigma_{x_{1}+x_{2}+\cdots+x_{n}} \sqrt{\frac{\alpha}{\alpha-2} t}
\end{aligned}
$$

$$
\text { for } n=2 \text { we put } \sum_{i=8}^{n} \prod_{i=2}^{i-1} \frac{i \alpha-2}{i \alpha-I}=0
$$

\begin{tabular}{|c|c|c|c|}
\hline$\alpha$ & 6 & Io & I 8 \\
\hline 2.5 & $0.814^{\circ}$ & 0.7679 & 0.7232 \\
\hline 3.0 & $0.7^{603}$ & 0.6545 & $0.592 \mathrm{I}$ \\
\hline 4.0 & 0.7085 & 0.5449 & 0.4676 \\
\hline \multicolumn{4}{|l|}{$n=3$} \\
\hline$\alpha$ & 6 & IO & 18 \\
\hline 2.5 & 0.8527 & 0.8034 & 0.7564 \\
\hline 3.0 & 0.8247 & 0.7038 & 0.6365 \\
\hline 4.0 & $0.797^{\circ}$ & 0.6050 & 0.5190 \\
\hline
\end{tabular}

Using the results from ExampleII we can calculate according to (37)

$\rho$ is a decreasing function in $t$, which means that the greater the expected number of claims the more independent the total loss becomes from the $n$ largest claims.

$\lim \rho(t)=0$

$t \rightarrow \infty$

$\rho$ is, of course, an increasing function of $n . \rho$ is decreasing in $\alpha$, i.e. the quicker the Pareto density function $f(x)=\alpha x^{-\alpha-1}$ converges to zero, the smaller becomes the contribution in percent of the $n$ largest claims to the total loss.

\section{Appendix:}

In analysing higher moments, formulae similar to $(28)$ could be derived. From (26), many formulae can be derived which might in certain cases be very important. 
I shall restrict myself here to a few examples.

a) Replace in (26) $\alpha$ by $\beta=\alpha^{2}, \alpha>\mathrm{I}$

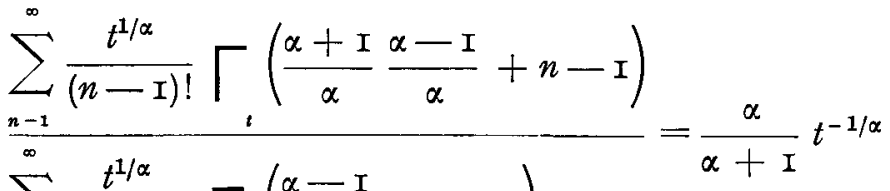

$$
\begin{aligned}
& \sum_{n=1}^{\infty} \frac{t^{1 / \alpha}}{(n-\mathrm{I}) !} \Gamma\left(\frac{\alpha-\mathrm{I}}{\alpha}+n-\mathrm{I}\right)
\end{aligned}
$$

b) Replace $\alpha$ by $\beta=\frac{1}{2}(\alpha+\mathrm{I})$ where $\alpha>$ I

$$
\sum_{n=1}^{\infty} \frac{t^{(2 / \alpha+1)}}{(n-\mathrm{I}) !} \Gamma\left(\frac{2 \alpha}{\alpha+\mathrm{I}}+n-2\right)=\frac{\alpha+\mathrm{I}}{\alpha-\mathrm{I}} t
$$

c) By partial integration we get:

$$
\begin{gathered}
\frac{\alpha}{\alpha-\mathrm{I}} t=\sum_{n=1}^{\infty} \frac{t^{1 / \alpha}}{(n-\mathrm{I}) !} \Gamma_{t}^{\left(\frac{n \alpha-\mathrm{I}}{\alpha}\right)=} \\
\sum_{n=2}^{\infty} \frac{t^{1 / \alpha}}{(n-\mathrm{I}) !} \frac{(n-\mathrm{I}) \alpha-\mathrm{I}}{\alpha} \Gamma_{t}\left(\frac{(n-\mathrm{I}) \alpha-\mathrm{I}}{\alpha}\right)- \\
\sum_{n=2}^{\infty} \frac{t^{n-1}}{(n-\mathrm{I}) !} e^{-t}+t^{1 / \alpha} \Gamma_{t}\left(\frac{\alpha-\mathrm{I}}{\alpha}\right) \\
\sum_{n=1}^{\infty} \frac{t^{1 / \alpha}}{(n-\mathrm{I}) !} \frac{n \alpha-\mathrm{I}}{n \alpha} \Gamma\left(\frac{n \alpha-\mathrm{I}}{\alpha}\right)=\frac{\alpha}{\alpha-\mathrm{I}} t+\mathrm{I}- \\
-e^{-t}-t^{1 / \alpha} \Gamma\left(\frac{\alpha-\mathrm{I}}{\alpha}\right)
\end{gathered}
$$

$\sum_{n=1}^{\infty} \frac{t^{1 / \alpha}}{n !} \Gamma\left(\frac{n \alpha-\mathrm{I}}{\alpha}\right)=\alpha\left[t^{1 / \alpha} \Gamma\left(\frac{\alpha-\mathrm{I}}{\alpha}\right)+e^{-t}-\mathrm{I}\right] \quad(26)-(40)$

$\sum_{n=1}^{\infty} \frac{t^{m / \alpha}}{n !} \Gamma\left(\frac{n \alpha-m}{\alpha}\right)=\frac{\alpha}{m}\left[t^{m / \alpha} \Gamma\left(\frac{\alpha-m}{\alpha}\right)+e^{-t}-\mathbf{I}\right]$ 
(27) and (42) can be used as upper and lower bounds of many similar sums with coefficients

$$
\frac{\mathrm{I}}{(n-\mathrm{I}) !}>C_{n}>\frac{\mathrm{I}}{n !}
$$

Example:

$$
\begin{gathered}
C \sum_{n=k-1}^{\infty} \frac{t^{2 / \alpha}}{n !} \Gamma\left(\frac{n \alpha-2}{\alpha}\right)<\sum_{t=k}^{\infty} E\left(x_{1} x_{n}\right)= \\
=t^{2 / \alpha} \sum_{n=k}^{\infty}\left[\prod_{i=1}^{n-1} \frac{\alpha}{i \alpha-I}\right] \Gamma\left(\frac{n \alpha-2}{\alpha}\right)<C \sum_{n=k-1}^{\infty} \frac{t^{2 / \alpha}}{(n-\mathrm{I}) !} \Gamma\left(\frac{n \alpha-2}{\alpha}\right)
\end{gathered}
$$

$C$ being a suitable constant for a given $\alpha$.

\section{LITERATURE}

[I] Franckx, E: Sur la fonction de distribution du sinistre le plus élevé. ASTIN Bulletin, Vol. II, Part III, I963.

[2] Ammeter, H.: Note Concerning the Distribution Function of the Total Loss Excluding the Largest Individual Claims. ASTIN Bulletin, Vol. III, Part II, 1964 .

3] Ammeter, H.: The Rating of "Largest Claim" Reinsurance Covers. Quarterly letter from the Algemeene Reinsurance Companies Jubilee Number 2, July I 964 . 